\title{
Autologous CMV-pp65-fILAMP mRNA Loaded Dendritic Cell Vaccine
}

National Cancer Institute

\section{Source}

National Cancer Institute. Autologous CMV-pp65-flLAMP mRNA Loaded Dendritic Cell

Vaccine. NCI Thesaurus. Code C156067.

A cancer cell vaccine consisting of autologous dendritic cells (DCs) loaded with mRNA encoding the human cytomegalovirus (CMV) matrix protein pp65 (65 kDa lower matrix phosphoprotein; UL83) as a fusion protein with the full-leng th lysosome-associated membrane protein (fILAMP), with potential immunostimulatory and antineoplastic activities. Upon vaccination, the autologous CMV-pp65-flLAMP mRNA loaded DC vaccine exposes the immune system to the CMV pp65 peptide, which may elicit a cytotoxic Tlymphocyte (CTL) response against CMV pp65-expressing tumor cells. The incorporation of fILAMP may route CMV pp-65 antigens into the lysosomal compartment, resulting in enhanced MHC class II antigen presentation, thereby promoting CD4-positive T-cell responses. The CMV pp65 protein is the primary component of the enveloped subviral particle of CMV and is expressed in certain tumor types. 